\title{
Mapping of Semantic Web Ontology in User Query System
}

\author{
Rupali R. Khune \\ Department of Computer \\ Engineering, MMCOE Pune, \\ University of Pune, India
}

\begin{abstract}
The main purpose of the Semantic Web promises a kind Machine Intelligence, which can support a verity of user tasks like efficiency of search engine or Question Answering (QA). Ontologies are needed for realization of the semantic web, which in turn depends on the ability of system to identify and take advantage of relationships that exist within ontologies. There are huge numbers of ontologies present on the web they need to be integrated for data integration. These ontologies are having different in representation, quality of data and larger sizes of ontologies, this lead to be problem during ontology mapping, on analyzing these problems and to introduce Multiagent mapping system. Main aim is to achieve heterogeneous data integration through semantic mapping of ontologies. this paper provide a mapping framework for Multiagent ontology having heterogeneous data in Semantic web and develop a question answering system from developed framework of ontology's and improve performance by adding semantic relation interpreter which improves response time.
\end{abstract}

\section{General Terms}

Ontology, semantic web, Similarity, multiagent.

\section{Keywords}

Automated question answering system, Multiagent System, Ontology Mapping, Semantic relation interpreter, semantic web

\section{INTRODUCTION}

We Web mining [1] uses data mining techniques to automatically discover and extract knowledge from web document, which can be in structured, unstructured or semi structured form. Semantic web means it provides the information which is understandable by Computer Machines. The aim semantic web [2] is to provide a common framework that allows data to be shared and reused across applications, so that data on the web is not just used for display purpose, but also make it understandable to machine so as to enhance the quality of information service and explore several new intelligent information services. Ontology as a set of knowledge terms, including the vocabulary, some simple rules of inference \& logic for some particular topic.

Ontology is basically description of key concept in given relationship including rules, properties, and concepts. It is the knowledge representation technology. Ontology is share a common understanding of structure among people or software agent. The main purpose of ontology is allowing user, to organize information of taxonomies according to their own attribute which describes the relationship between concepts or classes. Semantic web can be used to achieve mapping, matching and heterogeneous data integration. In recent years the development of number of Ontology mapping is first condition for achieving heterogeneous Data integration on semantic web. Do ontology mapping before data integration.
These Reflections lead us to ask how to understand the present Web and what developments anticipate. This is a deep question, and believes the history of science has something to teach us here. The semantic web is envisioned as a universal medium for data, information and knowledge exchange. With the ability of intelligent analyses, it can help people acquire appropriate information and discovery the latent semantic knowledge effectively.

More recently, areas that were once considered amenable only to analytic thought areas such as epistemology and logic are to some extent operational zed in computers and computer infrastructures. Knowledge representation and ontology engineering are about trying to capture aspects of shared conceptualizations; rapidly increasing semantic metadata on the Web will soon make it possible to develop efficient Semantic Web applications [2]. Ontology mapping in the QA system can provide more accurate results if the mapping process can deal with uncertainty effectively that is caused by the incomplete and inconsistent information used and produced by the mapping process. This is important because it will assure that more people will start using ontologies, which is initial condition for commercial viability of Semantic Web applications. The aim of the Semantic Web promises a kind of "machine intelligence," which can support a variety of user tasks like improved search or question answering (QA).

For such applications, researchers have developed a wide variety of building blocks that needs to be utilized to achieve wider public acceptance.[3] which makes it possible to interpret and align heterogeneous and distributed ontologies on the Semantic Web. However, to evaluate "machine intelligence" for ontology mapping, different problem have to be occurred. Consider, for example, the complexity of evaluating ontologies with a large number of concepts. Because the size of the vocabulary, a number of domain software agents are necessary to evaluate similar concepts in different ontologies. Once each agent has assessed sampled mappings, individual assessments are discussed, and a final assessment is produced, which result a collective judgment, aims at analysing the key trends and challenges of the ontology matching field. The main motivation behind this work is the fact that despite many component matching solutions that have been developed so far, there is no single solution to problem yet clear success, which is robust enough to be the basis for future development, and which is usable by non-expert users [4].There are various issues have to be posed during ontology mapping like these ontologies are different in size ,representation and various types of data, these problems are addressed in Multi-agent ontology mapping framework and solved by using Dempster theory of evidence .In this paper main contribution is to provide mapping framework for multi agent ontology having heterogeneous data in the semantic web and develop a question answering system from developed framework of ontology's. Semantic relation interpreter can be used to provide result in quick response 
time, which used compound nouns to analyse the semantic and syntactic similarity.

\section{RELATED WORK}

In literature survey, discuss recent methods over the Multiagent ontology mapping framework and compared with existing mapping systems.

N.Shadbolt,W.hall and T. Berners-Lee [2], the digital world has evolved at a prodigious rate. Today, the World Wide Web links 10 billion pages, and search engines can divine themes embodied in the links to serve useful and relevant content almost instantaneously. Yet today we believe that the Semantic Web is attain- able. We are seeing its first stirrings, and it will draw on some key insights, tools, and techniques derived from 50 years of $\mathrm{AI}$ research.

M. Nagy, M. Vargas-Vera, and E.Motta [3] Managing problems on the Semantic Web can accurately improve the ontology mapping precision that can be a better acceptance of systems operates in this environment. Another ontology mapping in the block of QA can provide more accurate results if the mapping process can deal with uncertainty effectively that is caused by the incomplete and inconsistent information used and produced by the mapping process. In this paper introduced algorithm called "DSSim " and describe the evaluation that had compared to OAEI 2006. State of ontology, purpose of ontology, general statement the problem of mapping two ontologies effectively and efficiently is a necessary precondition to integrate information on the Semantic Web. The proposed method usually combines syntactic and semantic measure by combining several techniques from heuristics to machine learning and also removes the uncertain reasoning.

P. Shvaiko and J. Euzenat [4], in this paper, they discussed ten challenges for ontology matching, accompanied for each of these with an overview of the recent advances in the field. They believe that challenges outlined are on the critical path; hence, addressing them should accelerate progress of ontology matching. Moreover, these challenges are not isolated from each other's: collaborative matching requires an alignment infrastructure; alignment evolution and other operations of alignment management require reasoning with alignments; user involvement would benefit from and contribute to collaborative matching; etc. Hence, these challenges, even if clearly identified will certainly have to be considered in prospective relation with each other.

Bock and Hettenhausen [5], in this paper, they has been designed to address the need for highly scalable, massively parallel tool for both large scale and numerous ontology alignments. It models ontology problem as an optimization prolem. It is especially suitable for providing answers under time constraint like the ontology mapping. Draw of this system is it did not participated in very large cross lingual resource track therefore experimental comparison cannot be achieved.

P.Wang and B. Xu [6], in this paper, they used semantic sub graph, semantic description document (SSD) for ontology alignment. Ontology mapping debugging is used to improve alignment result. The drawback of this system is it does not performed on relatively large size of tracks because of its multilingual representation. In this System this drawback is solved.

Y. R. Jean-Mary and M. R. Kabuka [7], in this paper, they developed an automated mapping tool for heterogeneous data integration using different matchers.It creates only pre alignment using the best values. Drawback of this system is did not combine the similarity because they consider only pre alignments. This drawback is over come in this system.

F. Hamdi, H. Zargayouna, B. Safar, and C. Reynaud [8], TaxoMap is an alignment tool; it is specially developed to retrieval useful alignments for data integration between different source ontologies. Drawback of this system is it does not process instances only consider hierarchically manner.

In proposed system WordNet uses as the background knowledge this ensures that this can provide equivalent mappings on different domains and domain independence.

From literature survey, summarize that system is performing well as compared to existing systems.

\section{IMPLEMENTATION DETAILS}

The proposed system aims to present and extend the existing methods and concept. Main objective is to provide a mapping framework [9] for Multiagent ontology having heterogeneous data in semantic web and develop a question answering system from developed framework of ontology's. For ontology mapping in the QA system over heterogeneous sources propose a multiagent framework because domains are large in size and more complicated, open and distributed, a set of cooperating agents are necessary in order to address the ontology mapping task. In real case scenarios, ontology mapping can be carried out on with huge number of classes and properties of domains.

Multiagent framework decrease response time when number of concept to map deceases. Provide an integrated ontology mapping framework to solve the different problems of integration. A solution called DSSim [3] which is prototype for the proposed architecture that combine with automated question answering system at the moment. In proposed system integrate multiagent system with the semantic relation interpreter of compound nouns, which operate effectively in Multiagent framework. Below Fig. 1 shows the proposed architecture of this system.

Input: User question set

Output: Set of answers generated by this framework

The proposed architecture is explained below in details.

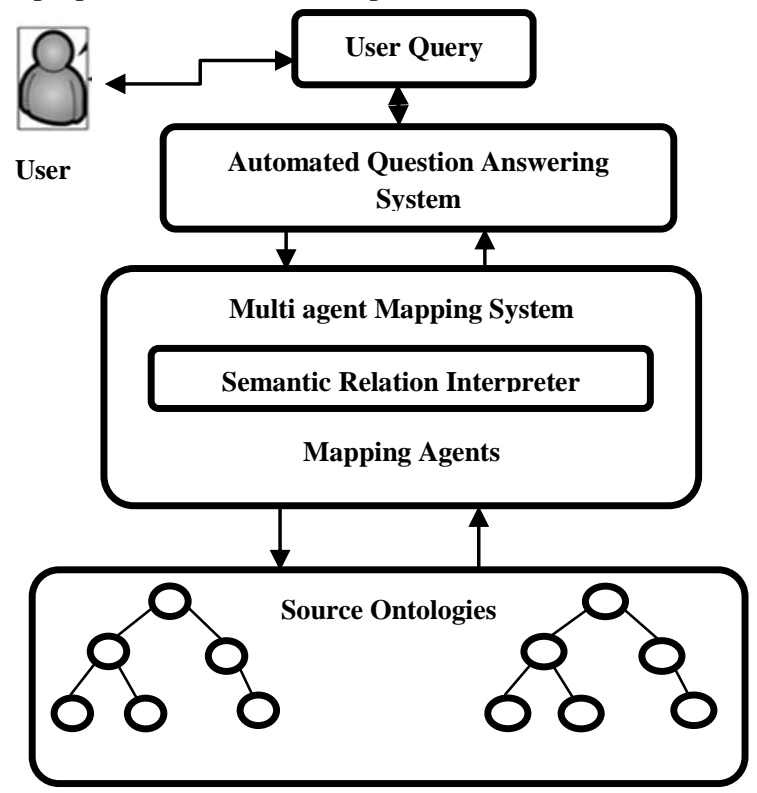

Fig.1 Proposed system 


\subsection{Ontology Mapping}

Ontology mapping captures relation between different ontologies, which increases precision value. Ontology mapping is pre condition for achieving heterogeneous data integration on the semantic web. Ontologies can be used as domain specific background knowledge by ontology mapping systems to increase the mapping precision.

Following Fig shows the ontology mapping between two ontologies such as Staff Ontology and Personnel Ontology. They are different in representation and size.

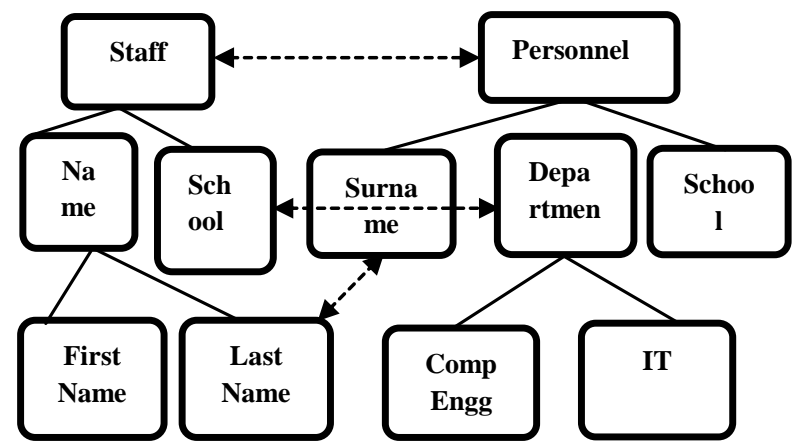

Fig. 2 Mapping Of Two Ontologies

\subsection{User Interface}

In this system used automated question answering System, which contains NLP techniques and Answer Consumption. This query interface is search engine sort type interface like Google or MSN. User poses a natural language query to the AQUA[10] System, which then converts user query into First Order Logic Terms. The AQUA System needs to create ontology mapping between both the concepts and properties of different domains and query terms posed by user.

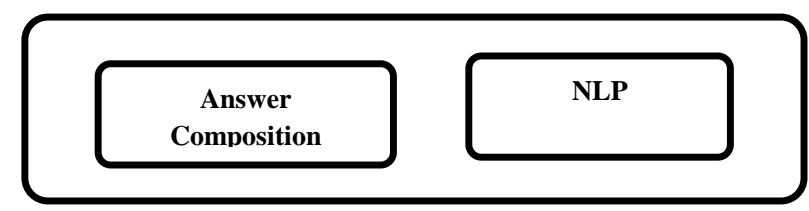

Fig.3 Question Answering System

NLP: Natural language processing is human readable and it can also be read by suitable software agent. Output of this component is logical representation of the query.NLP techniques provide accuracy in answers to user queries.

Answer Composition: Answer composition creates answer to user question, which is data provided by mapping agents through source ontologies and WordNet.

WordNet: It is lexical database for English language. It groups English words into set of synonyms called synsets. It is used as dictionary in Automated Question Answering System.

\subsection{Dempster-Shafer Theory and \\ Combination Rule}

In real cases the user poses different questions that contain both concepts and properties of particular domain. This information can be used to query the different ontologies, create mapping between its concepts and properties that can be used to answer particular user query take a concept (or Property) from ontology and consider it as the query fragment that would normally be posed by a user. From the query fragment build up a graph contains the close context of the query fragment such as the concepts and properties and its synonyms to the query graph from ontology and build a graph that contains both concepts and its synonyms. DempsterShafer Theory of evidence [11] which provides a mechanism for modelling and reasoning uncertain information in a numerical way, particularly when it is not possible to assign belief to a single element of a set of variables.

Consequently, the theory allows the user to represent uncertainty for knowledge representation, because the interval between support and plausibility can be easily assessed for a set of hypotheses. Ontology fragment need to define a reasonable limit on the number of synonyms, which are extracted from the WordNet. To define such a limit is also desirable when carry out the belief combination since all extracted terms represent a variable where each similarity value needs to be combined with the Dempster's rule of combination.

\subsection{Semantic Similarity and Syntactic Similarity Algorithms}

The Similarity algorithms are used to find quantitative similarity values between the nodes of the query and ontology fragment which is considered as an uncertain and objective assessment. For semantic similarity between concepts, relations and properties used graph based techniques like SimilarityBase Algorithm and SimilarityTop Algorithm [12] to find out most similar concepts/relation by using dice coefficient. For syntactic similarity [14] used string based techniques, to match names and name descriptions and also used edit distance function and jaccard[13] similarity.

\subsection{Semantic Relation interpreter}

In this process semantic relation interpreter of noun compounds which help to find out the semantic and syntactic similarity. This process uses binary selection feature extractor and selection methods to select particular similarity. similarity. This processs uses binary selection feature extractor and selection methods to select particular similarity.

\section{DATA SET}

Use Source ontologies to retrieve the similar content for ontology mapping. These ontologies are made up of standard data set, it is tree like structure. Mapping candidate can retrieve similarity from different nodes of ontology. Mapping can be done between different ontologies and integrate data from all these ontologies by using ontology mapping. Main aim is to achieve heterogeneous data integration. Use protégé software for ontology mapping.

WordNet is lexical Database for English language. It is one of the most widely used semantic resources in linguistics. It groups English words into sets of synonyms called synsets. Provide small general definition and records the various semantic relations between these synonym sets. WordNet play important role in this project because it consists of many synset related to this project.

\section{RESULT SET}

Each system is usually designed to address particular need from a specific domain. Compare this system with those are participated in OAEI(Ontology Alignment Evaluation Initiative) competitions. The evaluation uses Recall, Precision and F-measure, which are useful measure that have a fixed range. The parameters are important form mapping point of view. These parameters are considered for experimental analysis and for comparison with existing systems 
A. Precision: A measure of usefulness of hit list is an order list of hits in decreasing order of relevance to the query. Precision is calculated as

B. Recall: this is the completeness of hits measure and shows how better the performance of engine to finding relevant entities.

C. F-measure: the weighted harmonic means of precision and recall. Harmonic mean is used to calculate the average of a set numbers.

as compare with other sytem this system perform well.below graph shows the comparison result.

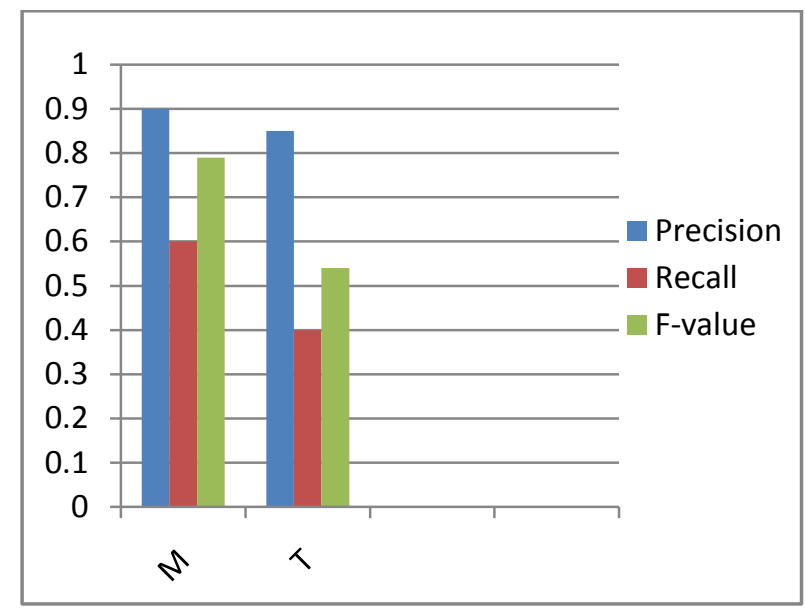

Fig 4.Excepted result as comparison with other system

\section{CONCLUSION}

In this paper, develop Multiagent ontology mapping framework, integrate this framework with automated question answering system, WordNet and source ontology. Automated question answering system used as user interface where user poses query and create answer to user query. This framework achieves heterogeneous data integration through ontology mapping. WordNet and standard source ontology's are used as a data set to retrieve data. Retrieve syntactic and semantic similarity for providing answer to user query. Semantic relation interpreter can be used to decreases the search time and improve the matching time. dempster Shafer theory used to solve the uncertain reasoning problem during ontology mapping. In this system quickly retrieve accurate result for user. The future scope is it can be used with different applications like Google search, research and matching accurate results in space related technologies in terms of similarity retrieve from different resource into integrated source'.

\section{REFERENCES}

[1] Wang Yong-gui JIA Zhen,"Research on Semantic Web Mining",IEEE 2010,vol 1,pp67-70.

[2] N. Shadbolt, W. Hall, and T. Berners-Lee, "The semantic web revisited,'IEEE Intell. Syst., vol. 21, no. 3, pp. 96 101, Jan./Feb. 2006.

[3] M. Nagy, M. Vargas-Vera, and E.Motta, "DSSimManaging uncertainty on the semantic web," in Proc. 2nd Int. Workshop Ontology Matching, 2007, pp. 156-168.

[4] P. Shvaiko and J. Euzenat, "Ten challenges for ontology matching," in Proc. OTM Confederated Int. Conf., 2008, pp. 1164-1182.

[5] Bock,J and Hettenhausen J.(2008) "MapPSO Result for OAEI 2008 "Processding of $3^{\text {rd }}$ International Workshop on Ontology Matching,Germany,Karlsruhe.

[6] M. Nagy, M. Vargas-Vera, and E.Motta, "DSSimManaging uncertainty on the semantic web," in Proc. 2nd Int. Workshop Ontology Matching, 2007.

[7] P.Wang and B. Xu, "Lily: Ontology alignment results for OAEI 2008," in Proc. 3rd Int. Workshop Ontology Matching, 2008, pp. 168-180.

[8] P. Lambrix and H. Tan, "SAMBO-A system for aligning and merging biomedical ontologies," J. Web Semantics, vol. 4, no. 3, pp. 196-206,2006.

[9] 26 M. Nagy, M. Vargas-Vera, and E. Motta, "Multiagent ontology mapping framework in the aqua question answering system," in Proc. $4^{\text {th }}$ MICAI-Advances in Artificial Intelligence, 2005, pp. 70-79.

[10] M. Vargas-Vera and E. Motta, "Aqua-Ontology-based question answering system," in Proc. 3rd MICAI, 2004 vol. 2972, pp. 468-477.

[11] G. Shafer, A Mathematical Theory of Evidence. Princeton, NJ: Princeton Univ. Press, 1976.

[12] M. Vargas-Vera and E. Motta, "An ontology-driven similarity algorithm,"Knowl. Media Inst., Open Univ., Milton Keynes, U.K., Tech. Rep.

[13] W. W. Cohen, P. Ravikumar, and S. E. Fienberg, "A comparison of string distance metrics for name-matching tasks," in Proc. IIWeb, 2003, pp. 73-78.

[14] A. E. Monge and C. P. Elkan, "The field matching problem: Algorithms and applications," in Proc. 2nd Int Conf. Knowl. Discov. Data Mining,1996, pp. 167-270. 